\title{
Research of bidding Control Strategy for Multi-agent Based Microgrid
}

\author{
Jian Luo, Jihong Shi, Jiang Yu, Yingli Xie \\ Department of Information Science and Engineering, Yunnan University, Kunming, 650091, China
}

\begin{abstract}
As a kind of power system, microgrid integrates the distributed power, the energy storage system and the controllable loads being connected to the low-voltage power grid. It can operate in the island-mode and connected-mode. In this paper, we focus on the study of the control strategy, take the reasonable assumption to design consumption schedule, and build a multi-agent microgrid architecture based on the Matlab and ZEUS platforms. Considering that the power supply capacity is insufficient to meet the electricity consumption demands for all loads, a real-time access control method based on the bidding control strategy is proposed. The effectiveness of the method is confirmed by the simulation.
\end{abstract}

\section{Keywords-microgrid; multi-agent; control strategy}

\section{INTRODUCTION}

Microgrid is a small power supply system which integrates the distributed energy, the energy storage system and the controllable loads being connected to the low-voltage power grid. As an application of distributed power generation, microgrid has the certain ability to independently supply the power, and it also can coordinate with the main electric grid. Besides, it has the properties of flexible application, changeable structure, and so on ${ }^{[1]}$. The distributed power generation technology, also known as decentralized generation technology, refers to generate electricity by using the photovoltaic, wind energy, bio-energy and other clean energy sources. This technology is characterized by the distribution around the user, less pollution, and electricity quality. Distributed generation units and the large electric grid can be as spare with each other through the grid-connected technique, which greatly improve the stability of power supply ${ }^{[2]}$. However, the control of microgrid is the core and difficult point of the microgrid technology, which mainly includes the switching of microgrid operation mode, the choice of the control theory of microgrid system, the control of the distributed power, load protection, and access control ${ }^{[3-5]}$. In this paper, a real-time access control method for loads according to the bidding control strategy is presented, and its effectiveness is validated by simulation based on Matlab and ZEUS platforms.

\section{Multi-AgENT MiCROGRID STRUCTURE AND COMMUNICATION MODE BASED ON ZEUS}

\section{A. ZEUS Platform}

ZEUS proposed by Intelligent Agent research group of British telecommunication laboratory is a design and development platform, which integrates the existing agent technical specifications for the rapid development of the collaborative multi-agent systems. By defining a standard set of single agent functional structure and multi-agent communication coordination system, ZEUS allows users to get rid of the complex and cumbersome definition work of agent underlying structure, and enables users to focus on the design and implementation of application problem and quickly develop the collaborative multi-agent application systems. ZEUS system strictly follows the FIPA 97 specifications, and all the Java source programs of the system are open to the users for further research and extension [6].

\section{B. Multi-agent System (MAS) Structure}

Microgrid belongs to the distribution network side of a main electric grid with respect to the distribution network. As a whole, microgrid can be viewed as a load of the distribution network. It can accept the control of distribution network, and also can provide its own operating parameters to the distribution network. Meanwhile, the lower layer of microgrid contains various components (distributed power, load, etc.). All kinds of components have its own independent running status and control mode. If the loads consume the electricity according to their actual situations, the distributed power will adjust the output based on the power consumption inside the electric grid. Therefore, a three-layer structure of distribution network consisting of agent, microgrid control agent, and component agent is proposed in this paper. The corresponding schematic diagram is shown in Fig.1.

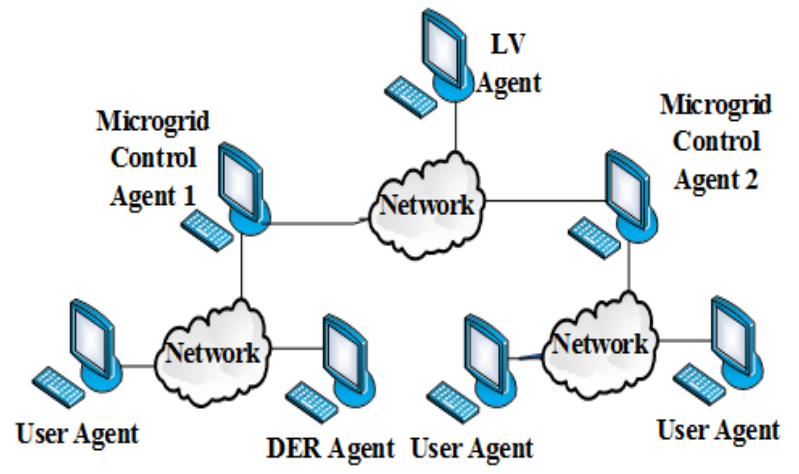

Figure 1. Schematic diagram of a three-layer structure of the distribution network 


\section{Microgrid Communication Mode on MAS Structure}

Different agents can communicate with each other to ensure the reasonableness of their decision-making. This kind of structure and communication mode can adapt the complex and flexible characteristics of the microgird. The communication mode of microgrid control system based on multi-agent mainly includes [7].Direct information transmission mode, that is direct information exchange of peer to peer or peer to group federal system communications and broadcast communications. Indirect information transmission mode, also called blackboard systems, namely, all the necessary information will be posted on a global database, and indirect information exchange on the same or similar levels of agent retrieves. This approach can provide sufficient data to agent for collaborative control.

\section{StRATEgy OF BIDDING CONTROL BASED ON MATLAB / SIMULINK AND ZEUS}

A prerequisite of bidding control strategy is that when the main electric grid fails, microgrid operates in island mode, and its supply capacity is insufficient to meet electricity demand for all critical/non-critical loads. Thus, there is a need to take some control strategies to control the load access. The bidding process is completed through the information exchange between the DER agents and the user agent. This process includes the following four steps: (1) bid initiated; (2) bid to buy; (3) bid acceptance; (4) operate non-critical loads. Fig. 2 shows the procedure and information exchange of a bidding control strategy.

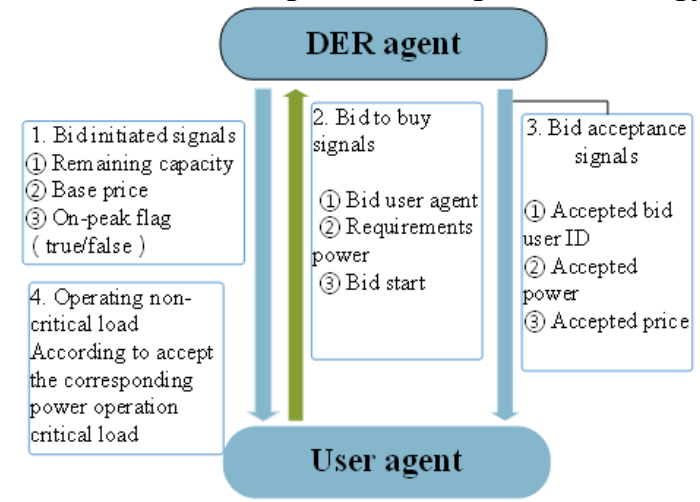

Figure 2. Procedure and information exchange of a bidding control strategy.

\section{A. DER Agent-bidding Initiated}

When the microgrid switches from the grid-connected mode to the island mode, the microgrid control agent firstly sends a broadcast signal to all agents to inform the current microgrid state. After user agent receives the broadcast signal, all the non-critical loads disconnected. DER agent will increase its power output to $50 \mathrm{kw}$ after receiving the broadcast signal, and inform the remaining capacity to all user agents in the grid through a bidding signal. The biding signal should include the following three contents: (1) Remaining capacity (kw); (2) Bidding base price (yuan/kwh); (3) On-peak-flag (true/false). In the control strategy, electricity tariff of all critical loads should be a pre-determined value, and the value is set to be the biding base price in the paper. Due to the loads are time-varying, the price should be periodically sent to all user agents after the island mode occurring.

Remaining capacity is the surplus of the power generation capacity after DER agent serves all of critical loads. Because the number of the critical load is timevarying, and user consumption schedule has been initialized. So the remaining capacity of each time period is calculated according to the user's consumption schedule.

Bidding base price is the lowest price of power resource which DER agent is willing to sell out. The price will be timely adjusted according to the consumption schedule, and it will increase with the reduction of the remaining power. In this paper, consumption schedule is divided hourly, so the bidding base price should be adjusted once per hour. The lowest and highest base price in the paper are defined as 0.5 yuan and 0.65 yuan, respectively.

On-peak-flag (true/false) represents the sign of the power consumption peak, which can be obtained by calculating the consumption schedule in each period time of the electricity/power ratio. When the ratio exceeds $80 \%$, the status of on-peak-flag is true. In such a case, user agent needs to bid for power use. Otherwise, the status becomes false, and the user consumes power according to the biding base price.

\section{B. User Agent-bidding to Buy.}

When the microgrid operates in the island mode, user agent firstly disconnects all the running non-critical loads [8], meanwhile, waiting for the bid signal of DER agent. When a required power user agent receives the DER bidding signal, it sents the buy signal to the DER agent,and the signal contains following three contents: (1) Power requirement (kw) ; (2) bid price (yuan / kwh) ; (3) User agent ID.

Power requirement: At the period time of microgrid switching to the island mode, the user agent corresponding to the non-critical loads which requires power will calculate the load demand power after receiving a bid signal.

Bid price: The user agent should do judgments to its on-peak-flag status after receives a bid signal. For different on-peak-flag status, there are different bidding prices.

When the status of on-peak-flag is false, it indicates that remaining capacity of power generation of DER agent at the current moment is abundant. And after providing all the critical loads, the capacity can still meet the power demands of all non-critical loads. So there is no power competition for all the power demands between the noncritical loads at present time, and user agent's bid price is the bidding base price of DER agent. When the status of on-peak-flag is true, it implies that the current time is the time of power use peak. At this moment, user agent's bid price should be higher than the base price since there is no 
enough remaining power to meet the electricity demands for all non-critical loads at same time. Bid price= Bidding base price + constant value, this constant value is set by the user. To reflect differences among user bidding prices, a array of constant value $[0.1,0.2,0.3,0.4,0.5]$ is defined, and all user agents randomly select the bid price from the array.

\section{DER agent-bidding acceptance.}

Receiving bid signals from various user agents, DER agent make the following judgments for each bid price:

When the bid price is lower than reserve price, DER agent reject to serve this user agent;

When the bid price is higher than reserve price, user agent with the highest price get the priority of power use agent, and the second highest one is the next;

When the bid price is higher than base price and two bidders give same price, user agent with demand for more power get the priority;

When the bid price is higher than base price, bid prices are identical and same power demanded, the user agent evenly allocates the remaining power.

After comparing bid prices, DER agent send bidding acceptance signal to all the bid to buy user agents. The signal contains following three contents: (1) ID of the accepted biding user; (2) accepted power (kw); (3) accepted price (yuan/kwh).

\section{User agent-operating non-critical load.}

After receiving the DER agent bid acceptance signal, user agent compares the received power and demand power of the DER.

When received power is equal to the demand power, user agent opens all its responsible non-critical loads.

When demand power is less than the received power, user agent opens the most significant load to provide power.

\section{SimUlation AND ANALYSIS OF THE CONTROL STRATEGY}

In the ZUES control interface, we should set the "Fault" and "Recover" buttons. When pressing the "Fault" button, the main electric grid fails, and microgrid switches into island mode. When pressing the "Recover" button, the failure of main electric grid is eliminated and microgrid switches into grid-connected mode. DER interface monitors DER breaker and DER power information. When the DER breaker status is false, it means that DER output power is $50 \mathrm{kw}$; When the DER breaker status is true, it indicates that DER output power is $25 \mathrm{kw}$. The status of each load switch is displayed in the user interface. True and false represent load consumption and disconnection, respectively. When the microgrid operates in the island mode, the user interface will display the bid price of each load.

\section{A. MATLAB Simulation of Microgrid.}

Electrical components of MATLAB/SIMULINK provide an excellent platform for the microgrid simulation, and the majority of current domestic and international studies on the microgrid are based on this platform. In order to facilitate research, we build a small microgrid architecture, as shown in Fig. 3

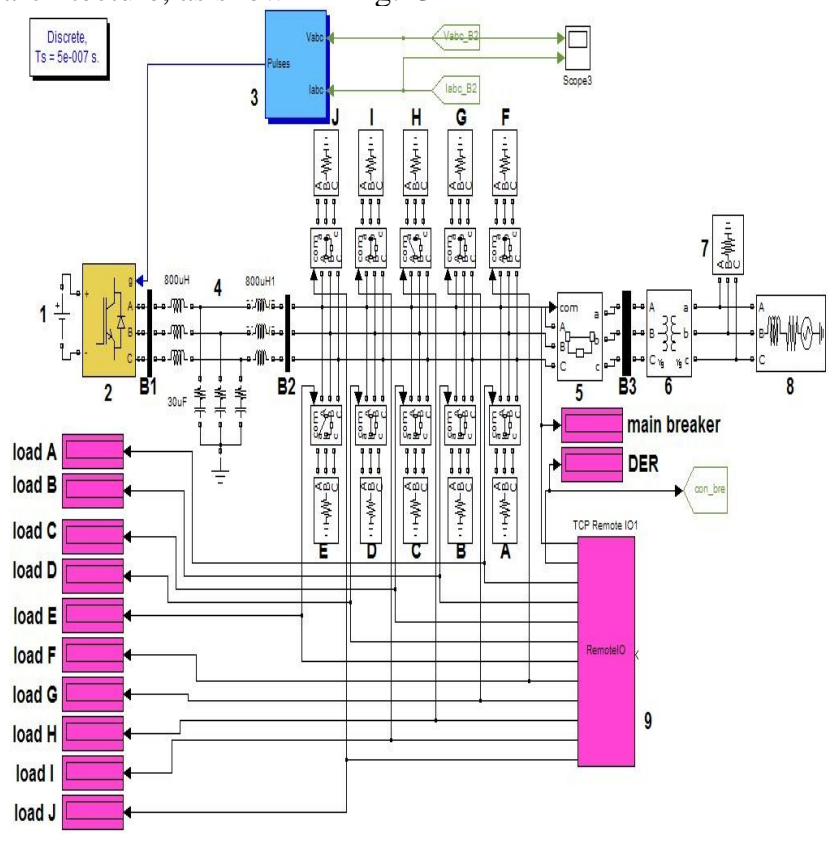

Figure 3. A small microgrid architecture

In Fig. 3, 1 is the distributed power section with capacity of $50 \mathrm{kw}$; 2 is an inverter for three-phase $220 \mathrm{~V}$ AC; 3 is inverter controller section, using pulse width modulation (PWM), by controlling inverter gate signal $\mathrm{g}$ to output the constant communication. Meanwhile, inverter controller connects to the upper layer control agent via the interface module, and receive the upper agent control decision signal, and use current tracking to control the inverter output power; B1, B2, B3 is the threephase voltage / current meter, which is used to monitor circuit voltage/current information; 4 is an LRC filter network for reduce harmonic interference; A-J is the 10 user loads, each load connect to microgrid through circuit breaker; 5 is main electric grid breaker for changing the operating mode by controlling the breaker on-off; 6 is the distribution network transformer; 7 is the load of distribution network; 8 is $10 \mathrm{kv}$ low-voltage distribution network; 9 is the TCP / IP component intermediate module, which is used to transmit 12 channels signals, and transfer control information of ZEUS agent to main electric grid breaker, DER and 10 users loads, and observe every signal external display component, respectively.

\section{B. Establishment of User Electricity Schedule.}

We divide a day into 24 time periods, and the load consumption on each period is relatively fixed for a practical microgrid. Assuming that the microgrid has "AJ" 10 loads, and its consumption scheme is shown in Table 1. 
TABLE I. A MiCROgRid CONSUMPTION SCHEDULE FOR 24 Hours

\begin{tabular}{|c|l|l|l|l|l|l|}
\hline \multicolumn{7}{|c|}{ (A B C D E F G H I J)=(5.4 6 6.2 6.5 5.7 6.8 7.2 6.6 6.4 5.2)kw } \\
\hline Time & $\begin{array}{l}\text { Critical } \\
\text { load }\end{array}$ & $\begin{array}{c}\text { Remain } \\
\text { power(kw) }\end{array}$ & $\begin{array}{l}\text { Non- } \\
\text { critical } \\
\text { load }\end{array}$ & $\begin{array}{l}\text { Consumption } \\
(\mathbf{k w})\end{array}$ & On_peak_flag & $\begin{array}{l}\text { Base price } \\
\text { (yuan/kwh) }\end{array}$ \\
\hline $1 \mathrm{am}$ & AB & 38.6 & CDEF & 36.6 & false & 0.5 \\
\hline $2-5 \mathrm{am}$ & AB & 38.6 & CD & 24.1 & false & 0.5 \\
\hline $6 \mathrm{am}$ & ABG & 31.4 & CD & 31.3 & false & 0.53 \\
\hline $7 \mathrm{am}$ & ABGH & 24.8 & CDEF & 50.4 & true & 0.54 \\
\hline $8 \mathrm{am}$ & ABGH & 24.8 & CDEFI & 56.8 & true & 0.54 \\
\hline $9 \mathrm{am}$ & ABFIJ & 20.2 & CDEGH & 62 & true & 0.58 \\
\hline $10-11 \mathrm{am}$ & ABFGIJ & 13 & CDEH & 62 & true & 0.65 \\
\hline $0 \mathrm{am}$ & ABFGI & 18.2 & CDEH & 56.8 & true & 0.6 \\
\hline $1-2 \mathrm{pm}$ & AB & 38.6 & CDEF & 36.6 & false & 0.5 \\
\hline $3 p m$ & ABFIJ & 20.2 & CDEHG & 62 & true & 0.58 \\
\hline $4-5 p m$ & ABFGI & 13 & CDEH & 62 & true & 0.65 \\
\hline $6 p m$ & ABFGI & 18.2 & CDEH & 56.8 & true & 0.6 \\
\hline $7 p m$ & ABGHIJ & 13.2 & CDEF & 62 & true & 0.63 \\
\hline $8-9 p m$ & ABHIJ & 20.4 & CDEFG & 62 & true & 0.56 \\
\hline $10-11 \mathrm{pm}$ & ABH & 32 & CDEFG & 50.4 & true & 0.52 \\
\hline $12 \mathrm{pm}$ & ABH & 32 & CDEF & 43.2 & true & 0.52 \\
\hline
\end{tabular}

From table 1, we can see that the critical loads and non-critical loads vary with time. For example, school bells are critical load during the day but non-critical loads at night. Microgrid DER in the paper has total generation capacity of $50 \mathrm{kw}$. The remaining power is the power left after supplying to the critical loads at each period of time, that is the total power can be distributed to all non-critical loads. Consumption contains the total power of the microgrid for the critical loads and the non-critical loads at each period of time. Status of on-peak-flag is used to indicate current time is peak period or not, and it can be determined by the ratio of the consumption and generation capacity. When the value larger than $80 \%$, the status is true, otherwise is false. When the bid base price is 0.50.65 yuan, it is set according to the remaining power.

C. Bid Simulation of Microgrid Switch Grid-connected Mode into Island Mode.

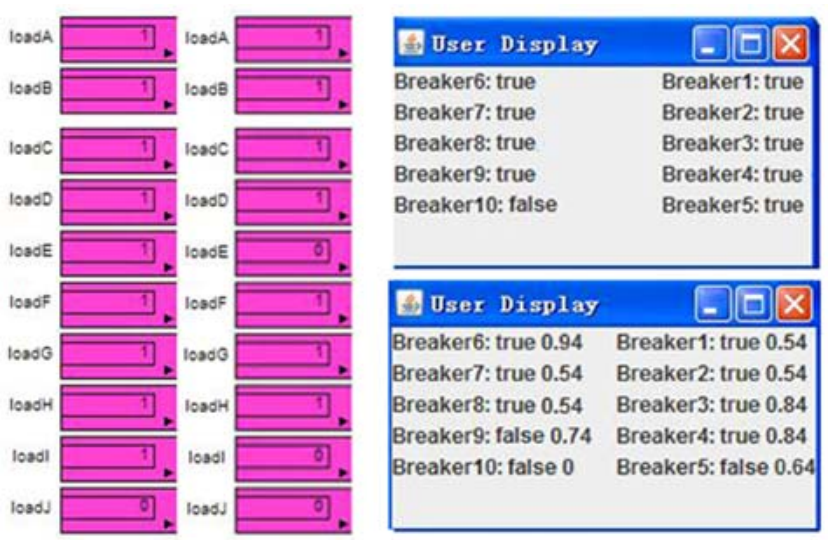

Figure 4. Bid simulation of Microgrid switching from grid-connected mode to the island mode
Based on the control strategy in this paper, in island mode, in order to ensure the critical load normally work, user agent should firstly disconnect all the non-critical loads to strictly protect critical loads electricity, and the loads accessing to the microgrid at this time are critical loads. As shown in consumption schedule, there is surplus power at this time, and start bidding to make non-critical loads access controlled by bid strategy. Bid simulation of microgrid switching from grid-connected mode to island mode is illustrated in Fig. 4.

In Fig. 4, left is comparison of load switch properties of MATLAB/SIMULINK, in which "1" and "0" represent switch on and off, respectively. Right is changes of load switch properties under ZEUS interface, which can be obtained through setting monitor user agent interface, and true/false represents the load access switch on/off. When microgrid fails at 8:00 am, user agent knows the critical load is $A B G H$ through checking user consumption schedule. And before receiving DER agent bid initiated signal, user agent will cut off all connection of noncritical loads. By checking the user consumption schedule, it is found that the electricity demands for the critical loads at period of 8:00 am are $\mathrm{ABGH}$, and non-critical loads are CDEFI. Therefore, when microgrid in the mode of grid-connected mode, load switches from A to I should be on, and load J have no consumption with switching on, which can be demonstrated in the simulation of Figure 4. When the main electric grid fails in a certain time between 8:00 to 9:00 am, after microgrid switches to island mode, each of agent coordinates communication in accordance with the rules, and initiate bidding. Based on bidding strategy, critical loads ABGH according to the current bid base price of 0.54 (yuan $/ \mathrm{kwh}$ ) for consumption, noncritical loads CDF according to bid price of [0.84, 0.84 , 0.94] (yuan/kwh) for consumption. We note that the total power of CDF loads are $19.5 \mathrm{kw}$. After DER provided the 7 loads, remaining power is only $5.3 \mathrm{kw}$, which is 
insufficient to supply loads E (5.7kw) and I (6.4kw) of bid price lower than CDF. Obviously, bidding strategy strict control the load access.

\section{V.CONCLUSIONS}

In this paper, we present a real-time access control method for loads based on the bidding control strategy. The load access status and bid prices at each period of time are obtained by comparing the simulation results with the electricity consumption schedule. The effectiveness and feasibility of proposed method are clearly verified.

\section{ACKNOWLEDGEMENTS}

The authors thank the Research Project from the National Natural Science Foundation of China "The key technology research of intelligent distribution network's collaborative communication based on IP and multiagent" (Grant NO.61162004) for financial support.

\section{REFERENCES}

[1] Che, Y.B. \& J.Chen. Research on Design and Control of Microgrid System. Journal of Przeglad Elektrotechniczny, 88(1), pp. 8386,2012.
[2] Nafar. M. Minimum Cost Design of Distributed Energy Resources with Studying the Effect of Capital Cost and Replacement Cost. Research Journal of Applied Sciences, Engineering and Technology, 4(3), pp.165-171,2012.

[3] Wang, J.L, Li X.M, Yang H.J \& S.L Kong. Design and Realization of Microgrid Composing of Photovoltaic and Energy Storage System. Journal of Energy Procedia, 12(1),pp. 1008-1014,2011.

[4] Xiao, Z, Li T.H, Huang M \& J.H Shi. Hierarchical MAS Based Control Strategy for Microgrid. Journal of Energy, 3(9), pp.16221638,2010.

[5] Wang, X.F, Guerrero J.M, Blaabjerg F \& Z. Chen. A Review of Power Electronics Based Microgrids. Journal of Power Electronics, 12(1), pp.181-192,2012.

[6] Collis, J.C, Ndumu D.T, Nwana H.S \& L. C. Lee. The ZEUS Agent Building Tool-kit. BT Technology Journal, 16(3), pp.60-68,2012.

[7] Wu. J. Research on Applications of Power Communication in Intelligent Distributed Network with Generation (The Master's Thesis of Shanghai Jiao Tong University, China 2010)

[8] Akbari, M, Moghaddas-Tafreshi S.M \& M. A. Golkar. WaveletBased Multi-Resolution Voltage Controller in a Hybrid AC/DC Microgrid. Journal of Przeglad Elektrotechniczny, 88(1), pp. 197204,2012 . 